\title{
Données préliminaires sur le paludisme humain en zones rurale et sémi-urbaine du département du Nkam (Littoral-Cameroun)
}

\author{
P. Ntonga Akono (1*), A. Mbida Mbida (1), C. Tonga (1), A. Yomon Kayoum (1), L. Enga Youmbi (1), L.G. \\ Lehman (1). \\ ${ }^{1}$ Laboratoire de Biologie et Physiologie Animale, Département de Biologie des Organismes Animaux, Université de \\ Douala, BP. 24157 Douala. \\ "Corresponding author : Laboratory of Biology and Animal Physiology, Department of Animal Biology, Faculty of Science, \\ University of Douala, P.O. Box 2701 Douala, Cameroon ; patakono2000@yahoo.fr. Tel. +237 697550847
}

Original submitted in on 20 $0^{\text {th }}$ April 2017. Published online at www.m.elewa.org on 31st July 2017

https://dx.doi.org/10.4314/jab/v115i1.4

\section{RESUME}

Objectif: Cette étude évalue les connaissances et les pratiques des populations sur le traitement et la prévention du paludisme et détermine la transmission du paludisme en zones rurale et sémi-urbaine du département du Nkam.

Méthodologie et résultats : Un questionnaire a été administré aux populations de Bodiman et Yabassi, localités rurale et semi-urbaine, en mai 2015. Les captures de moustiques sur volontaires ont été effectuées à l'intérieur de 7 habitations par site, de juillet à septembre 2015, au rythme de 2 nuits consécutives par mois. L'infectivité des vecteurs a été déterminée par le test ELISA-CSP. $73,17 \%(n=513)$ d'interviewés connaissaient les vecteurs du paludisme alors que seulement $9,46 \%(n=63)$ connaissaient le germe. La moustiquaire était l'outil de prévention le plus utilisé à Bodiman (100\%) et Yabassi $(38,46 \%)$. Le montant mensuellement dépensé par les ménages contre le paludisme s'élevait à 2,31€ et 16,01€ en moyenne, respectivement à Bodiman et Yabassi. La majorité d'interviewés à Bodiman $(n=98 ; \%=49,49)$ et Yabassi $(n=198 ; \%=42,31)$ se rendaient à l'hôpital en cas de paludisme. En outre, 37833 moustiques dont 35961 à Bodiman et 1872 à Yabassi ont été capturés. An. gambiae (81,82\%) et An. coluzzii (18,18\%) étaient les membres du complexe Gambiae identifiés ; An. coluzzii $(59,26 \%)$ étant le plus représenté à Yabassi, et $A n$. gambiae $(81,82 \%)$ à Bodiman. Les indices sporozoïtiques d'An. gambiae s.I. étaient de $0 \%$ à Bodiman et de $2,15 \%$ à Yabassi.

Conclusion et application : L'association de la lutte antilarvaire aux méthodes existantes de lutte antivectorielle par les pouvoirs publics contribuerait à baisser les fortes densités agressives observées à Bodiman de manière à réduire le budget alloué au paludisme par les ménages.

Mots-clés : Connaissances, pratiques, transmission, paludisme, Bodiman, Yabassi. 
Preliminary data on human malaria in rural and semi-urban areas of the Nkam division (LittoralCameroon)

\section{ABSTRACT}

Objectives: This study evaluates the knowledge and practices of the population in relation with to malaria prevention and treatment. It also determines malaria transmission in a rural and semi-urban settings of the Nkam division.

Methodology and results: A standard questionnaire was administered to populations of Bodiman and Yabassi, rural and semi-urban settings in May 2015. Mosquitoes were captured from July to September 2015, in 7 houses of each study site for 2 consecutive nights per month. The determination of the infectivity of the vectors was made by the ELISA-CSP test. In total, $73.17 \%(n=513)$ of interviewees knew malaria vectors whereas $9.46 \%(n=63)$ knew the germ. Mosquito net was the most used preventive tool in Bodiman (100\%) and Yabassi $(38.46 \%)$. The monthly amount spent per households for the prevention and treatment of malaria was estimated to $€ 2.31$ and $€ 16.01$ in Bodiman and Yabassi respectively. The majority of interviewees in Bodiman $(n=98 ; \%=49.49)$ and Yabassi $(n=198 ; \%=42.31)$ would go to the hospital in case of malaria. Overall 37833 mosquitoes were captured, 35961 in Bodiman and 1872 to Yabassi. An. gambiae (81.82\%) and Anopheles coluzzii (18.18\%) were the members of the Gambiae complex identified; An. coluzzii (59.26\%) was the most represented in Yabassi, and An. gambiae (81.82\%) in Bodiman. Sporozoitic rates of An. gambiae s. I. were $0 \%$ in Bodiman and $2.15 \%$ in Yabassi.

Conclusion and application of results: The implementation by public authorities of larval control in association with existing vector control methods would contribute in the reduction of aggressive mosquitoes density observed in Bodiman with subsequent reduction of malaria related expenditures of households.

Keywords: Knowledge, practices, transmission, malaria, Bodiman, Yabassi.

\section{INTRODUCTION}

Le paludisme demeure la maladie parasitaire la plus meurtrière au monde (OMS, 2014). Les données statistiques révèlent entre 473000 et 789000 , le nombre de décès dus à cette maladie par an (OMS, 2014). Les enfants de moins de 5 ans et les femmes enceintes constituent les tranches de la population les plus vulnérables. Au Cameroun, cette maladie est responsable de $23 \%$ de cas d'hospitalisation et consomme en moyenne $40 \%$ du budget de santé de ménages (MINSANTE, 2008). Le paludisme apparaît de ce fait comme un problème majeur de santé publique au Cameroun. Depuis des décennies, le Cameroun déploie d'énormes moyens pour lutter contre le paludisme. Cette lutte est principalement axée sur le diagnostic précoce et la prise en charge rapide des infections avec des combinaisons thérapeutiques à base d'artémisinine, la prophylaxie chez les femmes enceintes avec la sulfadoxinepyriméthamine et la lutte intégrée contre les vecteurs avec une composante de lutte chimique basée sur les aspersions intra-domiciliaires et la distribution massive de moustiquaires imprégnées (Toto et al., 2011). Bien que les informations relevées ces deux dernières années aient montré une baisse de la densité anophélienne et de la prévalence paludique nationale, il convient de noter que la situation du paludisme demeure critique dans certaines faciès éco-climatiques de ce pays d'Afrique centrale. Ceci serait dû à l'uniformisation des mesures de lutte antipaludique sur toute l'étendue du territoire national sans tenir compte des spécificités écologiques et ethno-tribales rencontrées au Cameroun. En effet, le Cameroun présente un caractère climatique hétérogène. Cette hétérogénéité du climat est à l'origine de grands ensembles écologiques définissant chacun une modalité particulière de la transmission du paludisme. Par ailleurs, ce pays est un regroupement de plus de 250 ethnies vivant en harmonie et qui sont chacune intimement liée à ses pratiques et coutumes. Le plus souvent, la stratégie de lutte recommandée n'est pas toujours acceptée 
par certaines ethnies si celle-ci n'est pas compatible à leurs coutumes. Bien plus, les études menées dans certains villages au Cameroun n'ont montré que les moustiquaires destinées à se protéger des piqûres de moustiques étaient utilisées à des fins piscicoles (Nelson, comm. Pers.). Dans ce contexte, les acteurs de lutte antipaludique devraient prendre acte de telles disparités afin de définir de nouvelles approches en adéquation avec les réalités écologiques et ethno-tribales de chaque localité. Au Cameroun, le faible niveau de réussite des opérations de lutte en cours est en partie dû à la rareté des informations sur la dynamique de transmission du paludisme et la conception sociologique de cette parasitose par les populations dans plusieurs localités ; lesquelles informations sont susceptibles d'amener les acteurs de lutte à réviser leur stratégie de lutte de manière à ce que celle-ci soit en adéquation avec les spécificités locales. Bodiman et Yabassi, localités rurale et sémi-urbaine

\section{MATERIEL ET METHODES}

Sites d'étude: L'étude a été menée dans le Nkam $\left(5^{\circ} 10^{\prime} \mathrm{N} ; 11^{\circ} 10^{\prime} \mathrm{E}\right)$, l'un des 4 départements de la région $\mathrm{du}$ littoral (Cameroun). Le Nkam est bâti sur une circonférence superficielle de $6380 \mathrm{Km}^{2}$ et est peuplé d'environ 96979 habitants. Le climat est de type équatorial humide caractérisé par une grande saison des pluies qui s'étend du mois de Mars à celui de Novembre et une courte saison sèche qui va de Décembre à Février. Les précipitations moyennes annuelles sont de $2927 \mathrm{~mm}$ et la température moyenne annuelle est de $27,2^{\circ} \mathrm{C}$. Le réseau hydrographique est dense, principalement constitué de nombreux ruisseaux se déversant dans le fleuve Nkam. L'étude a précisément eu lieu dans deux sites choisis pour leurs particularités écologiques, Bodiman et Yabassi. Yabassi est situé à une centaine de kilomètres à l'Est de la ville de Douala. La végétation primitivement forestière, s'est progressivement dégradée avec l'urbanisation. Elle est formée d'espèces végétales telles que les Euphorbiacées, les Ulmacées, les Pipéracées et les Graminées. Le milieu naturel est de type semi-urbain. Les routes principales et secondaires sont non bitumées et parsemées de nombreux nids de poules. Depuis de nombreuses décennies, la ville connaît un brassage de populations provenant des 4 coins du pays. Celles-ci sont regroupées autour de plusieurs secteurs d'activité notamment l'agriculture, l'élevage, la pêche, la menuiserie, etc. Le réseau hydrographique est situées dans le département du NKAM font partie des localités du pays ne disposant pas encore de telles informations. Pourtant, ce département fait l'objet depuis quelques années d'une forte anthropisation suite à la mise en place d'infrastructures scolaires, universitaires et sanitaires sans oublier la création de vastes plantations à l'effet de répondre aux besoins alimentaires sans cesse croissants des populations. La mise en place de ces structures a entraîné un bouleversement important de l'environnement pouvant avoir un impact sur le comportement des vecteurs, la transmission du paludisme et par là même, les pratiques des populations pour se protéger des piqûres de moustiques ou traiter le paludisme. La présente recherche investigue sur les connaissances, attitudes et pratiques sur le traitement et la prévention du paludisme et détermine le niveau de transmission du paludisme en zones rurale et sémiurbaine du département du Nkam (Cameroun).

formé du fleuve Nkam, de nombreux ruisseaux, de marais et marécages; ceux-ci constituent avec les nids de poules, des gites potentiels pour les larves de moustiques. Bodiman, localité très peu anthropisée est situé à quelques encablures de la ville de Yabassi. Le milieu naturel est de type rural. La végétation est fermée, constituée de grandes essences d'arbres dont les cimes forment un couvert végétal empêchant un ensoleillement maximal des gittes de moustiques. Les habitations, faites en planches sont de type traditionnel. Les habitants utilisent les cuvettes, les fûts et autres récipients pour recueillir l'eau de pluie en saison pluvieuse, le village ne bénéficiant pas encore d'une adduction en eau potable. Le fleuve Nkam, les récipients à eau et quelques collections d'eau de surface rencontrées dans le village constituent des gîtes propices au développement des larves de moustiques. Les populations constituées majoritairement de l'ethnie Ewodi vivent de la pêche et de l'agriculture.

Recueil des informations: Les informations ont été recueillies aux mois de Mai 2015 à Bodiman et Yabassi à l'aide d'un questionnaire standard administré aux chefs de ménages de 4 quartiers recensés à Bodiman (Bonanyamsi, Bwemba, Bonandjeng et Dibon) et Yabassi (Bonabeke, Yabassi-Centre, Ndogbele, et Bonalembe). Après consentement libre et éclairé, les chefs de ménage ont été interviewés par une équipe de 5 personnes 
formées. L'interview se déroulait en privé à l'effet d'éviter l'influence des autres membres de la famille, en langues locale, française ou anglaise. II était principalement question de se renseigner (i) si les populations connaissent les causes du paludisme, (ii) si elles connaissent les gîtes larvaires des moustiques, (iii) sur les méthodes utilisées pour se prémunir des piqûres de moustiques, (iv) sur le budget alloué par chaque ménage pour le contrôle des vecteurs, (v) sur le budget mensuel alloué par chaque ménage pour le traitement du paludisme et (vi) sur la source de traitement du paludisme.

Échantillonnage et traitement des moustiques agressifs à l'homme : L'enquête a été menée avec l'autorisation de l'autorité préfectorale locale et l'accord des occupants des maisons prospectées. Ces maisons ont été choisies en fonction de leur proximité aux gîtes permanents de moustiques, dans les quartiers Yabassicentre, Bonabeke (Yabassi) et Dibon, Bonanyamsi,
Bwemba (Bodiman). L'échantillonnage des moustiques a été réalisé par la méthode de captures nocturnes sur volontaires humains soumis à une prophylaxie à travers l'administration per os de 3 comprimés de sulfadoxinepyriméthamine $500 \mathrm{mg} / 25 \mathrm{mg}$. Les captures ont eu lieu à l'intérieur de 7 habitations par site, de juillet à septembre 2015, au rythme de 2 nuits consécutives par mois (figure 1). Les moustiques étaient capturés à l'aide des tubes à hémolyse à la lueur de la lampe torche. Les collectes de chaque maison de capture ont été groupées par tranches horaires dans des sacs numérotés. Les moustiques collectés ont été morphologiquement identifiés sous une loupe binoculaire suivant les clés d'identification morphologique (Edwards, 1941 ; Gillies et De Meillon, 1968; Gillies et Coetzee, 1987). Les moustiques appartenant au genre Anopheles ont été placés dans des tubes Eppendorf tapissés au préalable de silicagel et ont ensuite été conservés dans un congélateur à $-20^{\circ} \mathrm{C}$ pour les analyses ultérieures.

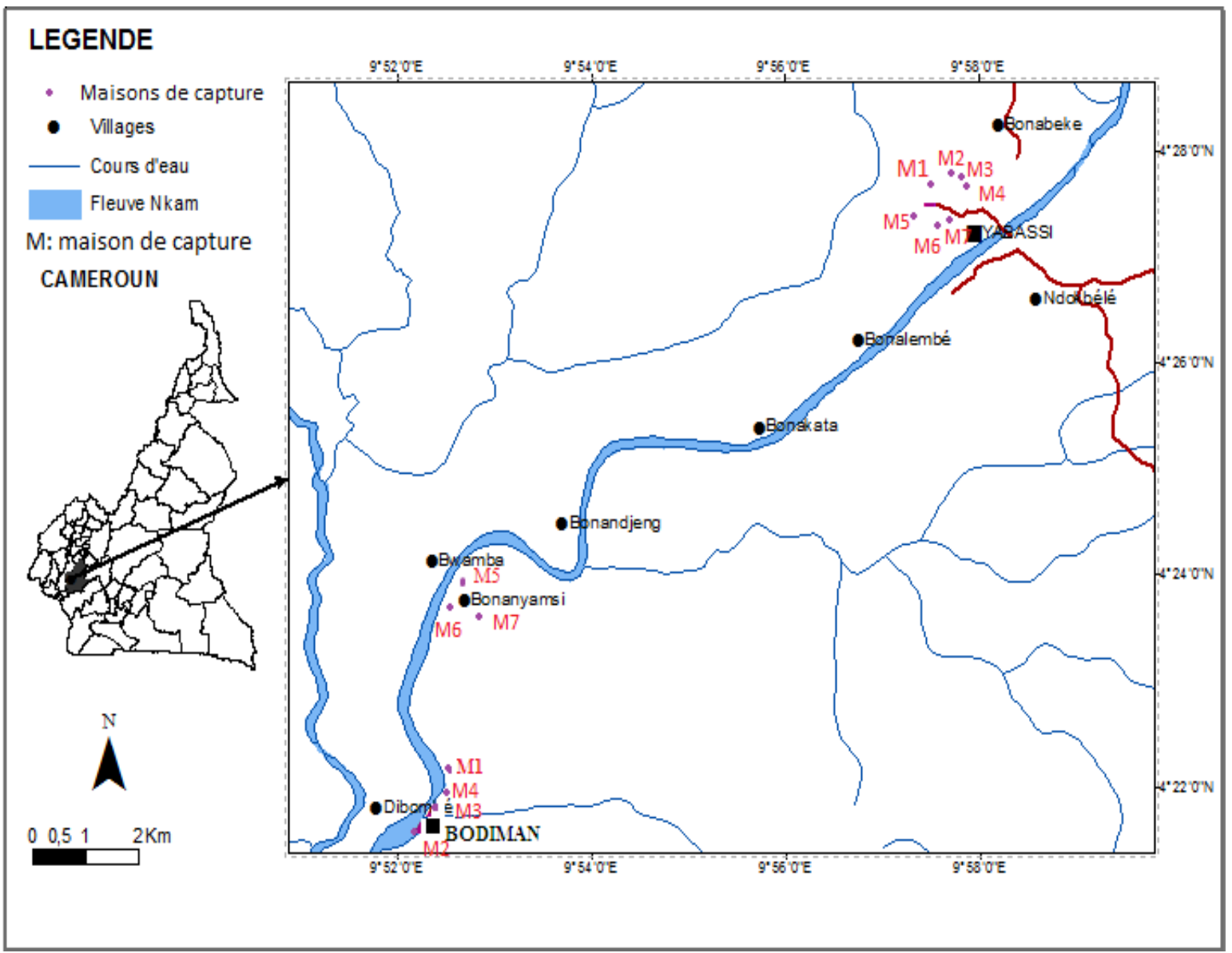

Figure1 : Localisation des maisons de captures des moustiques dans les sites d'étude 
Analyses moléculaires de laboratoire: La détermination des taux d'infection a été faite par détection de la protéine circumsporozoïte (CSP) à l'aide de la technique ELISA (Burkot et al., 1984). Les espèces et les formes moléculaires du complexe An. gambiae ont été identifiées par la PCR (Santolamazza et al., 2008).

Analyses statistiques des données

Calcul des indices entomologiques de la transmission : Le taux d'agressivité (m.a) est le nombre de piqûres qu'une personne reçoit d'une espèce vectrice par nuit. Il est exprimé en piqûres par Homme et par nuit

\section{RESULTATS}

Caractéristiques socio-démographiques : Un total de 666 chefs de ménages a accepté participer à cette étude, sur les 891 sollicités dans les deux sites, soit un taux de $(\mathrm{p} / \mathrm{H} / \mathrm{n})$. Le taux d'infection est la proportion de moustiques d'une espèce donnée, porteurs de sporozoïtes dans leurs glandes salivaires. Le taux d'inoculation entomologique (TIE) est le nombre de piqûres infectantes reçues par homme et par nuit. II est exprimé en piqûres infectées par Homme et par nuit (pi/H/n).

Tests de comparaison: Les tests de Mann-Whitney, khi-deux, Student et Fisher ont permis de comparer les différentes données. Le logiciel SPSS est celui utilisé.

participation de $74,74 \%$. Le nombre total de chefs de ménages interviewés était de 468 à Yabassi et 198 à Bodiman (tableau 1).

Tableau 1 : Connaissances, pratiques et niveau de dépenses des populations de Bodiman et Yabassi dans la prévention et le traitement du paludisme /

\begin{tabular}{|c|c|c|c|c|}
\hline Catégories & Caractéristiques & Yabassi & Bodiman & Total \\
\hline Ménages prospectés & Questionnés & 468 & 198 & 666 \\
\hline \multirow[t]{2}{*}{ Vecteurs du paludisme } & Oui & $387(82,70 \%)$ & $126(63,63 \%)$ & $513(77,02 \%)$ \\
\hline & Non & $81(17,30 \%)$ & $72(36,37 \%)$ & 153(22,98\%) \\
\hline \multirow[t]{2}{*}{ Germe du paludisme } & Oui & $54(11,54 \%)$ & $9(4,54 \%)$ & $63(9.46 \%)$ \\
\hline & Non & $414(88,46 \%)$ & $189(95,46 \%)$ & $603(90,54 \%)$ \\
\hline \multirow[t]{2}{*}{ Gîtes larvaires } & Oui & $369(78,84 \%)$ & $108(54,54 \%)$ & $477(71,62 \%)$ \\
\hline & Non & $99(21,16 \%)$ & $90(45,46 \%)$ & $189(28,38 \%)$ \\
\hline \multirow[t]{7}{*}{ Prévention } & Assainissement & $18(3,84 \%)$ & $0(0 \%)$ & $18(2,70 \%)$ \\
\hline & MILDA utilisées & $180(38,46 \%)$ & $198(100 \%)$ & $378(56,75 \%)$ \\
\hline & Aérosol & $0(0 \%)$ & $0(0 \%)$ & $0(0 \%)$ \\
\hline & Serpentin & $81(17,30 \%)$ & $0(0 \%)$ & $81(12,16 \%)$ \\
\hline & Méthodes combinées & $108(82,69 \%)$ & $0(0 \%)$ & $108(16,21 \%)$ \\
\hline & Aucune mesure & $54(11,53 \%)$ & $0(0 \%)$ & $54(8,11 \%)$ \\
\hline & Autres & $27(5,76 \%)$ & $0(0 \%)$ & $27(4,05 \%)$ \\
\hline \multirow[t]{2}{*}{ Origine des MILDA } & Don & $297(63,46 \%)$ & $63(31,81 \%)$ & $360(54,05 \%)$ \\
\hline & Achat & $18(3,84 \%)$ & $135(68,19 \%)$ & $153(22,97 \%)$ \\
\hline \multirow{4}{*}{$\begin{array}{l}\text { Causes de non utilisation des } \\
\text { MILDA }\end{array}$} & Chaleur & $9(1,92 \%)$ & $0(0 \%)$ & $9(1,35 \%)$ \\
\hline & Pêche & $101(21,58 \%)$ & $0(0 \%)$ & $101(15,16 \%)$ \\
\hline & Détériorées & $25(5,34 \%)$ & $0(0 \%)$ & $25(3,75 \%)$ \\
\hline & Non reçu & $153(32,69 \%)$ & $0(0 \%)$ & $153(22,97 \%)$ \\
\hline \multirow[t]{2}{*}{ Dépenses (FCFA) } & Contrôle du vecteur : & $730,769 \pm 205,900$ & $2305 \pm 736,545$ & $3035,769 \pm 942.4$ \\
\hline & $\begin{array}{l}\text { Traitement du } \\
\text { paludisme : }\end{array}$ & $5557,692 \pm 1011,123$ & $15450 \pm 2659,120$ & $21007 \pm 3670.2$ \\
\hline \multirow[t]{6}{*}{ Lieu de traitement } & Hôpital & $198(42,31 \%)$ & $98(49,49 \%)$ & $296(44,44 \%)$ \\
\hline & Pharmacie & $36(7,69 \%)$ & $25(12,63 \%)$ & $61(9,16 \%)$ \\
\hline & Médicament de la rue & $36(7,69 \%)$ & $8(4,04 \%)$ & $44(6,6 \%)$ \\
\hline & Médecine traditionnel & $9(1,92 \%)$ & $35(17,68 \%)$ & $44(6,6 \%)$ \\
\hline & Méthodes combinées & $99(21,15 \%)$ & $32(16,16 \%)$ & $131(19,67 \%)$ \\
\hline & Aucune mesure & $90(19,23 \%)$ & $0(0 \%)$ & $90(13,51 \%)$ \\
\hline
\end{tabular}

MILDA : Moustiquaires Imprégnées à Longue Durée d'Action 
Connaissances sur le paludisme et son vecteur : La majorité des enquêtés $(n=513 ; \%=77,02)$ dans les 2 sites ont établi un lien étroit entre les piqûres de moustiques et le paludisme. Ce taux était de $63,64 \%$ et de $82,69 \%$ respectivement à Bodiman et Yabassi. En revanche, très peu d'enquêtés $(n=63 ; \%=9,46)$ dans les deux sites connaissaient le germe du paludisme soit $4,54 \%$ de personnes interviewées à Bodiman et $11,54 \%$ à Yabassi. Le nombre d'enquêtés connaissant l'agent causal du paludisme était significativement inférieur à celui ne le connaissant pas $(p<0,001)$. Cependant, les niveaux de connaissance de l'agent causal du paludisme dans les deux sites d'étude n'étaient pas significativement différents $(p=0,666)$. La plupart de personnes enquêtées dans les sites d'étude $(n=477 ; \%=71,62)$ connaissaient les gites larvaires de moustique, soit $78,85 \%(n=369)$ à Yabassi et $54,55 \%(n=108)$ à Bodiman. Le niveau de connaissances des gîtes larvaires de moustiques par les personnes enquêtées à Yabassi était significativement supérieur à celui de personnes enquêtées à Bodiman $(p=0,003)$. Les méthodes préventives utilisées contre les piqûres de moustiques étaient: l'assainissement de l'environnement $(n=18 ; \%=27,02)$, l'utilisation des moustiquaires $(n=207 ; \%=31,08)$, l'utilisation des serpentins ( $n=81 ; \%=12,16)$, l'utilisation des méthodes combinées ( $n=306 ; \%=45,94)$ (tableau 1). Seulement 27 personnes enquêtées n'utilisaient aucune méthode préventive. La proportion de personnes interviewées utilisant les moustiquaires à Yabassi $(38,46 \%)$ était significativement supérieure à celle interviewée à Bodiman $\quad(13,63 \%) \quad(p<0,0001)$. La plupart des moustiquaires utilisées $(n=360)$ étaient des MILDA qui représentent un don des pouvoirs publics camerounais. Par ailleurs, $1,35 \%$ de personnes enquêtées associaient la non-utilisation des moustiquaires à la chaleur alors que $15,16 \%$ de personnes justifiaient ce manquement à l'utilisation des moustiquaires à des fins piscicoles (tableau 1).

Gestion des cas de paludisme : Près de la moitié des personnes enquêtées $(\%=45,31)$ se rendaient à l'hôpital en cas de paludisme. Cette proportion était de $42,31 \%$ $(n=198)$ à Yabassi et de $31,81 \%(n=63)$ à Bodiman. Ces proportions étaient significativement différentes dans les deux sites $\left(X^{2}=7,636 ; p=0,006\right)$. Les autres sources de traitement sollicitées par les enquêtés étaient la pharmacie, les médicaments de rue et les remèdes traditionnels (tableau 1).

Dépenses mensuelles liées au contrôle et au traitement du paludisme: Les enquêtés dépensaient en moyenne $2305 \mathrm{~F} \mathrm{CFA} \pm 736,545(3,51 € \pm 1,12)$ pour le contrôle du vecteur du paludisme à Bodiman contre 730,769 F CFA $\pm 205,9(1,1 € \pm 0,31)$ à Yabassi. Pour ce qui est des charges moyennes mensuelles liées au traitement, elles étaient de $15450 \mathrm{~F}$ CFA $\pm 2659,12$ $(23.55 € \pm 4.05)$ et de $5557,692 \mathrm{~F} \mathrm{CFA} \pm 1011,123(8,47 €$ $\pm 1,54$ ) respectivement à Bodiman et Yabassi (tableau 1). Diversité des moustiques agressifs à l'Homme: Au total, 37833 moustiques ont été capturés sur volontaires humains en 84 hommes-nuits de capture dans les 2 sites, dont 1872 à Yabassi et 35961 à Bodiman. 8 espèces de moustiques dont 3 anophèles ont morphologiquement été identifiées dans les deux sites d'étude (tableau 2). A Bodiman, un homme recevait $856,21 \mathrm{p} / \mathrm{h} / \mathrm{n}$. Culex poicilipes $(814,36 \mathrm{p} / \mathrm{h} / \mathrm{n})$ était l'espèce la plus agressive, suivie de Cx. quinquefasciatus ( $23,14 \mathrm{p} / \mathrm{h} / \mathrm{n})$, An. nili s.l. $(15,57 \mathrm{p} / \mathrm{h} / \mathrm{n})$, An. gambiae s.l. (2,36 p/h/n) et An. ziemanni $(0,79 \mathrm{p} / \mathrm{h} / \mathrm{n})$. A Yabassi, le taux global d'agressivité était de $44,57 \mathrm{p} / \mathrm{h} / \mathrm{n}$. L'espèce la plus agressive était $A n$. gambiae s.l. $(22,21 \mathrm{p} / \mathrm{h} / \mathrm{n})$, suivie de $C x$. quinquefasciatus (17,93 p/h/n), Cx. poicilipes (2,86 p/h/n), An. ziemanni $(0,64 \mathrm{p} / \mathrm{h} / \mathrm{n})$, An. nili s.l. $(0,36 \mathrm{p} / \mathrm{h} / \mathrm{n})$, Ae. aegypti $(0,36$ $\mathrm{p} / \mathrm{h} / \mathrm{n}), \mathrm{Cx}$. tigripes $(0,14 \mathrm{p} / \mathrm{h} / \mathrm{n})$ et Ae. albopictus $(0,07$ $\mathrm{p} / \mathrm{h} / \mathrm{n}$ ). L'identification moléculaire de 522 spécimens (261 spécimens de chaque site) du complexe Gambiae a montré qu'An. gambiae $(81,82 \%)$ était significativement plus fréquente qu'An. coluzzii $(18,18 \%)$ à Bodiman $(p<$ 0,0001 ). Par contre à Yabassi, les proportions de ces espèces cryptiques étaient presque semblables ( $p=$ $0,174)$. 
Akono et al., J. Appl. Biosci. 2017 Données préliminaires sur le paludisme humain en zones rurale et sémiurbaine du département du Nkam (Littoral-Cameroun)

Tableau 2 : Diversité culicidienne dans les localités de Bodiman et Yabassi

\begin{tabular}{|c|c|c|c|c|c|c|}
\hline \multirow[t]{2}{*}{ Espèces } & \multicolumn{2}{|c|}{ Yabassi } & \multicolumn{2}{|c|}{ Bodiman } & \multicolumn{2}{|l|}{ Total } \\
\hline & $\mathrm{n}$ & $\%$ & $\mathrm{n}$ & $\%$ & $\mathrm{n}$ & $\%$ \\
\hline An. gambiae s.l & 933 & 49,84 & 99 & 0,28 & 1032 & 2,73 \\
\hline An. nili s.l. & 15 & 0,80 & 654 & 1,82 & 669 & 1,77 \\
\hline An. ziemanni & 27 & 1,44 & 33 & 0,09 & 60 & 0,16 \\
\hline Cx. quinquefasciatus & 753 & 40,23 & 972 & 2,7 & 1725 & 4,55 \\
\hline Cx. Poicilipes & 120 & 6,41 & 34203 & 95,11 & 34323 & 90,72 \\
\hline Cx. tigripes & 6 & 0,32 & 0 & 0 & 6 & 0,02 \\
\hline Ae. albopictus & 3 & 0,16 & 0 & 0 & 3 & 0,01 \\
\hline Ae. Aegypti & 15 & 0,80 & 0 & 0 & 15 & 0,04 \\
\hline Total & 1872 & 100 & 35961 & 100 & 37833 & 100 \\
\hline
\end{tabular}

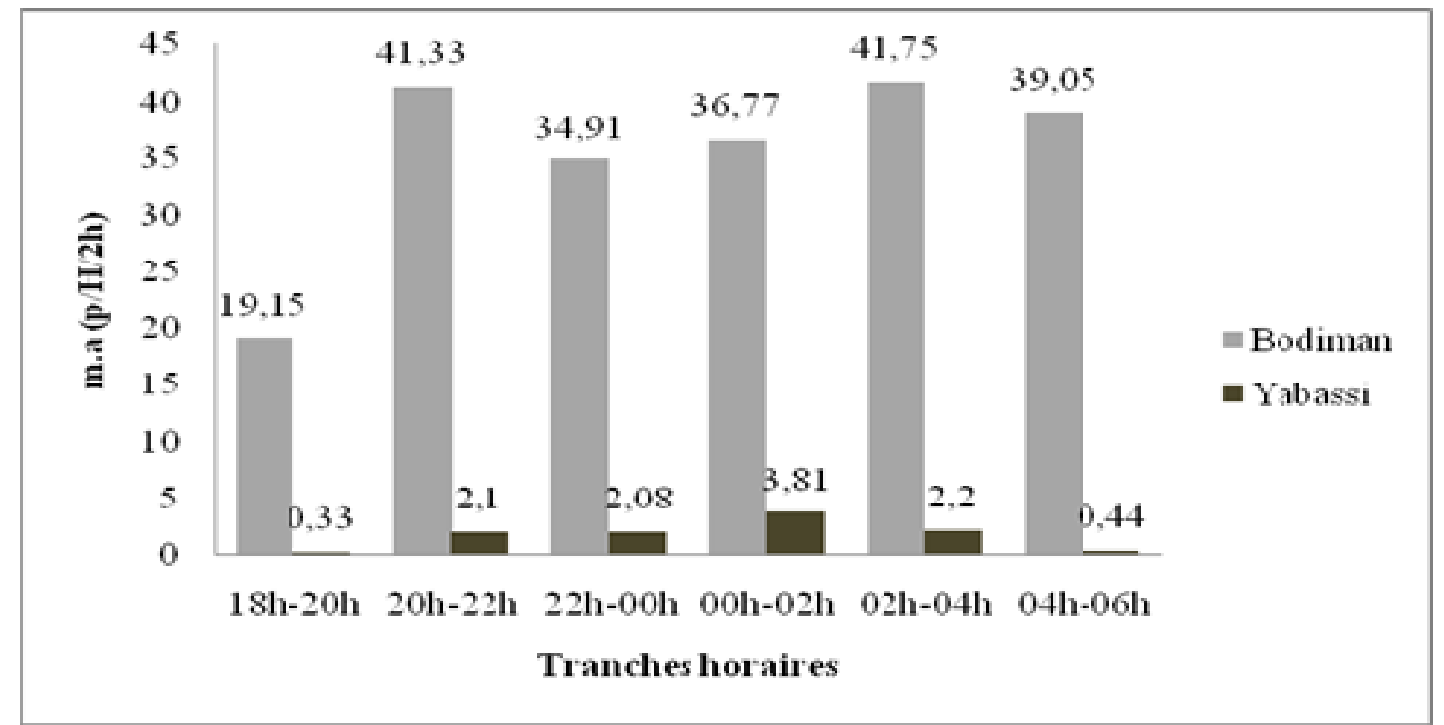

Figure 2 : Cycle d'agressivité globale des moustiques dans les sites d'études

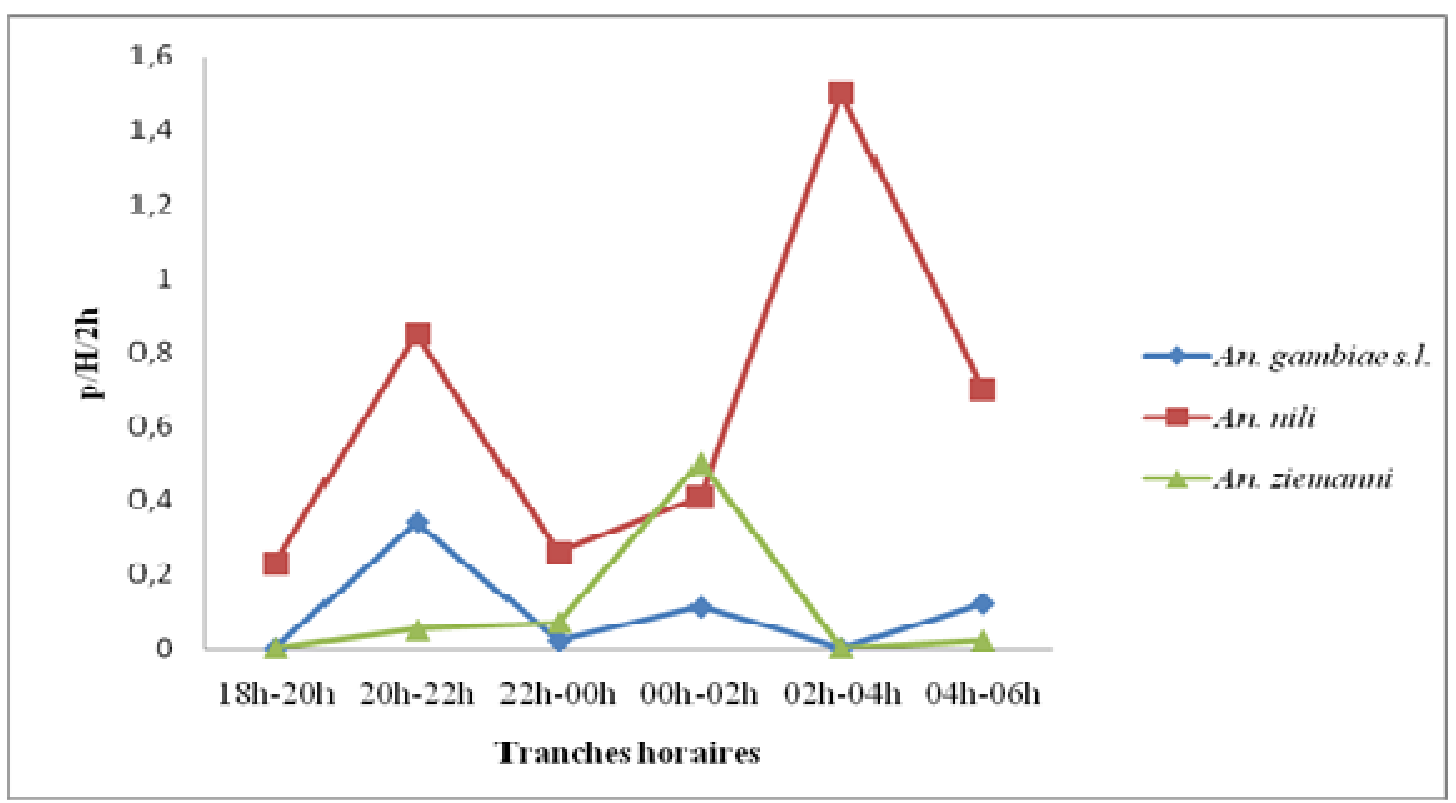

Figure 3 : Cycle d'agressivité des anophèles à Bodiman 


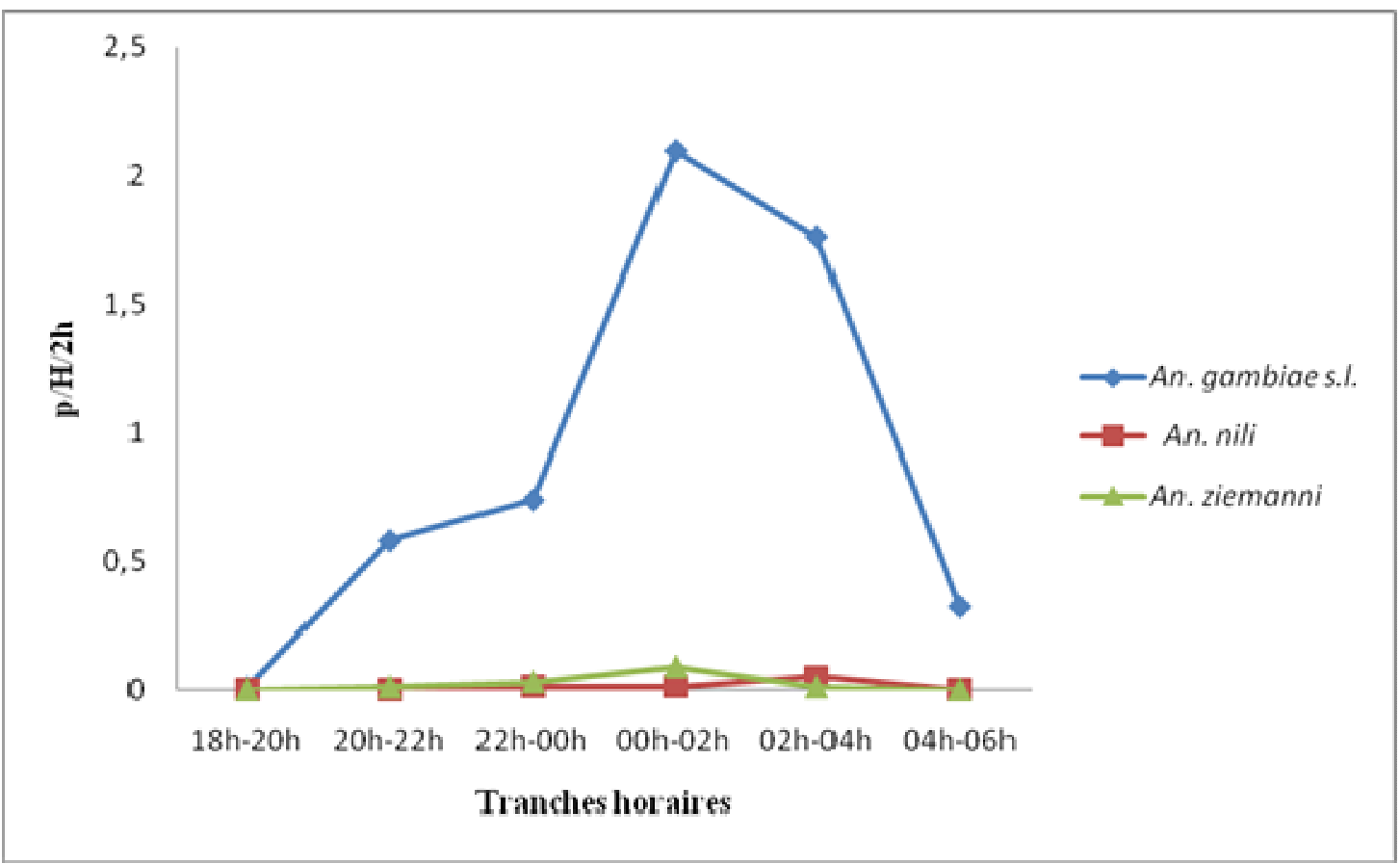

Figure 4 : Cycle d'agressivité des anophèles à Yabassi

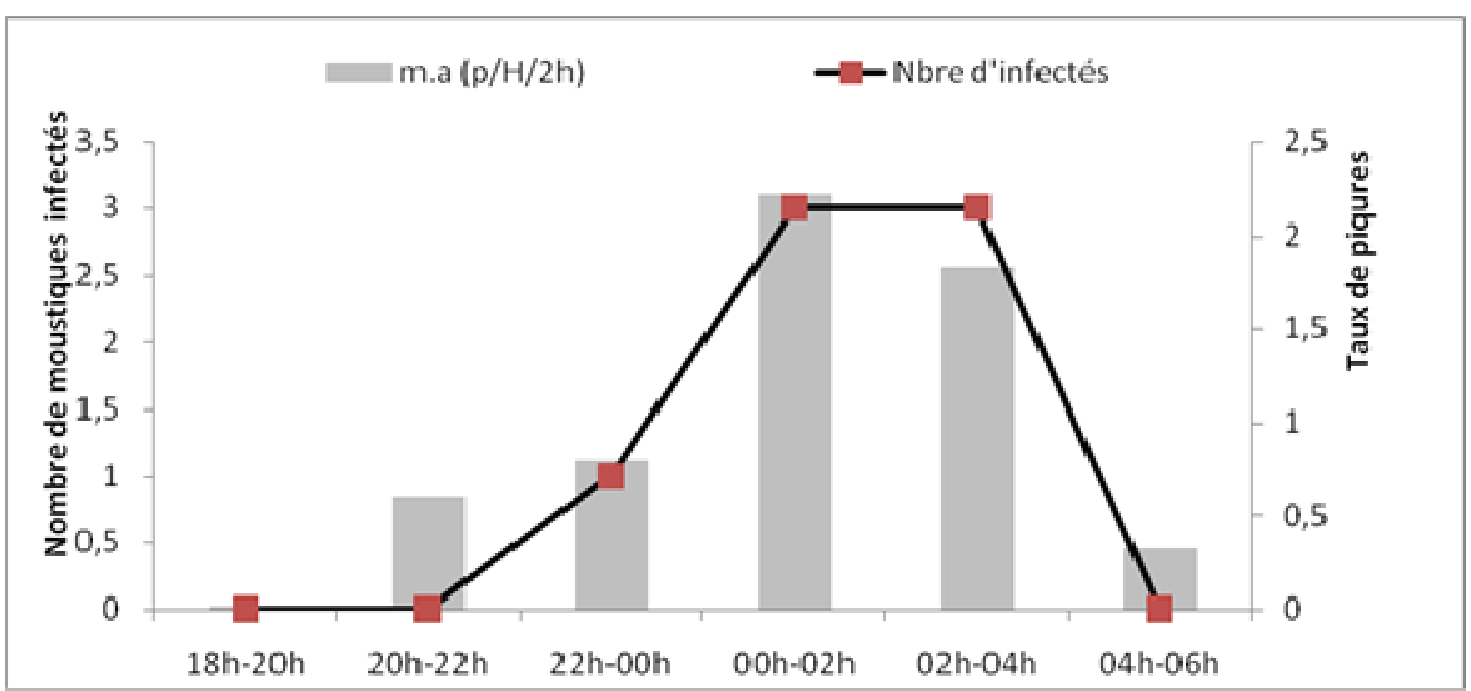

Figure 5 : Cycle d'agressivité et répartition horaire des femelles infectées d'An. gambiae s.I. à Yabassi/

Cycle d'agressivité : A Bodiman, le cycle d'agressivité globale des moustiques présente 2 pics : l'un entre $20 \mathrm{~h}$ $22 \mathrm{~h}$ et l'autre entre $2 \mathrm{~h}-4 \mathrm{~h}$. A Yabassi par contre, le cycle d'agressivité globale des moustiques croît progressivement et atteint un seul pic autour de 00h-02h puis décroît le reste de la nuit jusqu'à $6 \mathrm{~h}$ du matin (figure 2). Le cycle d'agressivité des anophèles à Bodiman est résumé dans la figure 3 . Celui d'An. nili présente deux pics dans les tranches $20 \mathrm{~h}-22 \mathrm{~h}$ et $2 \mathrm{~h}-4 \mathrm{~h}$ alors que les femelles d'An. gambiae s. I. sont plus agressives dans la tranche 20h-22h. A Yabassi, les femelles d'An. gambiae s.l. ont présenté une forte activité agressive dans la tranche $00 \mathrm{~h}-02 \mathrm{~h}$ alors que celles d'An. nili ont été plus agressives entre 02h-04h (figure 4).

Infectivité des vecteurs et transmission du paludisme : Au total, 1761 têtes-thorax des femelles d'anophèles dont 977 provenant de Yabassi et 784 de Bodiman ont été soumis au test ELISA-CSP. 21An. 

urbaine du département du Nkam (Littoral-Cameroun)

gambiae s.l. dont 12 An. gambiae et 9 An. coluzzii provenant tous de Yabassi étaient infestées par $P$. falciparum. L'indice sporozoitique (Is) moyen était de 2,15\% à Yabassi et de $0 \%$ à Bodiman. Les femelles d'An. gambiae s.l. infectées ont été capturées dans les

\section{DISCUSSION}

La présente étude a montré une similarité du niveau de connaissances des vecteurs du paludisme et leurs gîtes de ponte par les populations dans les sites enquêtés. $\mathrm{Ce}$ résultat corrobore celui enregistré dans l'aire de santé de Baguinéda au Mali dans laquelle les populations des villages enquêtés incriminaient dans leur grande majorité les moustiques comme la principale cause du paludisme (Goita, 2010). Cependant, le niveau de connaissance de l'agent causal du paludisme par les enquêtés des deux localités est très faible. Ceci pourrait s'expliquer par la forte campagne de distribution de masse des MILDA en vigueur de nos jours au Cameroun. Les tapages médiatiques accompagnant cette campagne semblait indiquer que la lutte contre cette parasitose passait prioritairement par le contrôle des vecteurs reléguant au dernier rang les actions qu'on pouvait mener contre le germe. Malgré ce faible niveau de connaissance du germe relevé dans les sites d'étude, notons tout de même que les populations de Yabassi connaissaient mieux le germe du paludisme que celles de Bodiman. Ce résultat serait le fait de la différence du niveau de scolarisation des enquêtés. En effet, les personnes interrogées à Yabassi avaient un niveau de scolarisation plus élevé que celles de Bodiman. Cette situation se justifie par la présence de plusieurs infrastructures scolaires et académiques à Yabassi malheureusement absentes à Bodiman. Une analyse similaire a été faite au Sénégal (Seck et al., 2008). La MILDA était l'outil de prévention le plus utilisée dans les sites d'étude. Ce résultat s'expliquerait par les campagnes de distribution gratuite de cet outil de lutte anti-vectorielle au Cameroun d'abord en 2011 puis en 2015. En effet, la plupart des enquêtés vivaient en deçà du seuil de pauvreté et préféraient par conséquent recourir aux méthodes anti-vectorielles peu coûteuses voire gratuites. Bien plus, comparativement à d'autres outils, la MILDA présente cet avantage d'avoir une longue durée d'action et constitue à l'heure actuelle l'arme la plus indiquée pour se protéger des piqûres de moustiques (Carnevale et Mouchet, 2001 ; Lindsay et al., 1989 ; Lines et al., 1987). La MILDA était utilisée dans tous les ménages recensés à Bodiman alors que seulement $38,46 \%$ de ménages l'utilisaient à Yabassi. Cette différence d'utilisation serait imputable à la différence des taux d'agressivité enregistrée dans les tranches horaires $22 \mathrm{~h}-00 \mathrm{~h}, 00 \mathrm{~h}-02 \mathrm{~h}$ et $02 \mathrm{~h}-04 \mathrm{~h}$ (figure 5). La transmission du paludisme était assurée à Yabassi pendant la période d'étude par An. gambiae et $A n$. coluzzii. Le taux d'inoculation entomologique (TIE) dû à ces espèces anophéliennes était de $0,5 \mathrm{pi} / \mathrm{H} / \mathrm{n}$.

deux sites. En effet, la densité culicidienne agressive était 20 fois plus importante à Bodiman $(856,21 \mathrm{p} / \mathrm{h} / \mathrm{h})$ qu'à Yabassi $(44,57 \mathrm{p} / \mathrm{h} / \mathrm{n})$. Face à une telle agressivité culicidienne, la MILDA apparaît comme l'outil le plus indiqué pour se protéger des piqûres de moustiques. Les moustiquaires étaient utilisées en association avec les répulsifs (serpentins et bombes aérosols) dans certains ménages enquêtés dans les sites d'étude. Cette association trouve sa justification en ceci que les moustiques peuvent aussi piquer en dehors des heures de sommeil (Rickenbach et al., 1972); en plus la MILDA est susceptible de perdre sa toxicité au fil du temps. Ainsi, ces répulsifs seraient non seulement des outils de protection en dehors des heures de sommeil, mais aussi ceux qui visent à renforcer l'effet chimique des moustiquaires (Kelani et al., 2014). L'assainissement de l'environnement s'est avéré comme la méthode préventive la moins pratiquée par les enquêtés. Ceci serait dû aux défaillances relevées au sein du système éducatif local qui accorde moins de crédits à l'apprentissage des règles d'hygiène. Cette situation a forcément des répercussions sur la manière selon laquelle l'environnement est assaini. La source de traitement la plus sollicitée était l'hôpital alors que la moins sollicitée était les remèdes traditionnels. De plus en plus, on assiste à un regain de confiance des populations africaines à la médecine conventionnelle par rapport à celle traditionnelle. Ce regain de confiance résulterait des défaillances relevées dans la médecine traditionnelle qui, bien qu'étant efficace pèche par le non-respect des doses. La culicidofaune agressive à l'Homme est riche et diversifiée dans les sites d'étude, constituée de huit espèces. La prédominance de l'espèce $C x$. poicilipes à Bodiman pourrait s'expliquer par la présence autour des maisons d'habitations des marais encombrés de végétations, des fossés herbeux et des marécages. Cette écologie de l'espèce Culex poicilipes est semblable à celle décrite par Hamon et al. (1955). Les résultats montrent par ailleurs une présence du complexe Anopheles gambiae aussi bien à Yabassi qu'à Bodiman. La forte présence de ce complexe à Yabassi pourrait s'expliquer par une anthropisation croissante du milieu primitivement forestier. En effet, l'activité dévastatrice des exploitants forestiers, doublée de la mise en place 

urbaine du département du Nkam (Littoral-Cameroun)

d'infrastructures socio-éducatives et la création de vastes plantations ont mis en place de nombreuses flaques d'eaux stagnantes ensoleillées propices à la ponte et au développement des larves d'Anopheles gambiae. A Bodiman le caractère perméable du sol est à l'origine de la rareté des collections d'eaux stagnantes. Les quelques collections d'eau visibles ne sont pas ensoleillées compte tenu de l'aspect fermée que présente la forêt dans cette localité. Ce qui expliquerait la faible densité de ce complexe d'espèces dans cette localité. Cependant, cette localité montre une forte présence de l'espèce An. nili comparativement à Yabassi. Ce résultat est dû à la proximité du village Bodiman au fleuve Nkam. De nombreux auteurs ont montré que les larves d'An. nili se développent préférentiellement aux bords des cours d'eau à débit rapide (Carnevale et al., 1992). La présence d'An. ziemanni à Bodiman pourrait également être liée au fleuve Nkam et au visage forestier que présente la localité de Bodiman, An. ziemanni étant signalé par de nombreux auteurs comme étant une espèce forestière (Tabue et al., 2014). Culex quinquefasciatus, qui s'adapte mieux dans les gîtes pollués est l'espèce du genre Culex la plus représentée à Yabassi. Ce résultat corrobore ceux d'une étude faisant de cette espèce l'une des premières causes de nuisance culicidienne dans la plupart des villes d'Afrique (Antonio-Nkondjio et al., 2012). Les résultats des tests moléculaires effectués sur An. gambiae s.l. ont révélé qu'An. gambiae Giles et An. coluzzii Coetze et Wilkerson sont les seuls membres du complexe gambiae dans les sites d'étude. Ces résultats corroborent ceux enregistrés en zones forestières du Cameroun faisant de ces espèces des membres du complexe gambiae en zone forestière (Tene Fossog et al., 2015). A Bodiman, An. gambiae est l'espèce dominante du complexe Gambiae. Cette prédominance d'An. gambiae, au détriment d'An. coluzzii s'expliquerait par le visage plus naturel et donc moins pollué de ce village. A Yabassi, la prédominance de l'espèce $A n$. coluzzii serait dû à la multiplicité des gîtes larvaires saumâtres. Ces résultats sont en accord avec ceux de certains auteurs qui ont signalé qu'An. coluzzii est une espèce qui s'adapte mieux aux eaux saumâtres de surface (Etang et al., 2016). Bien

\section{CONCLUSION}

La présente étude a montré une faible connaissance de l'agent causal du paludisme ainsi que des voies peu recommandées utilisées par une tranche de la population pour se soigner des affections liées à cette parasitose dans les sites prospectés Des campagnes de sensibilisation devraient être menées à l'effet d'amener plus, Yabassi est une zone semi-urbaine présentant un niveau de pollution nettement plus élevé que Bodiman. Ces résultats sont en accord avec ceux qui avaient signalé l'adaptation des stades pré-imaginaux d'An. coluzzii aux polluants (Tene Fossog et al., 2013). L'étude du cycle nocturne d'agressivité des Culicidae a montré des pics d'activité situés entre 00h-02h à Yabassi et 02h04h à Bodiman. A Yabassi, le pic est atteint dans la tranche horaire 00h-02h pour An. gambiae s.l. Ce résultat est similaire à ceux trouvés dans plusieurs régions du Cameroun (Antonio-Nkondjio et al., 2012 ; Akono et al., 2015). A Bodiman, le pic d'activité d'An. gambiae s.l. est situé dans la tranche $20 \mathrm{~h}-22 \mathrm{~h}$. Ce résultat corrobore celui ayant montré que le pic d'agressivité d'An. gambiae s.l. était précoce dans les maisons dotées de moustiquaires imprégnées de Deltaméthrine à Djoumouna au Congo (Zoulani et al., 1994). Les tests ELISA-CSP ont montré que l'indice sporozoïtique est nul à Bodiman. Ce résultat serait dû à la faible densité de la population humaine dans cette localité et à l'usage généralisé des moustiquaires qui réduirait fortement les contacts Homme/vecteurs. En effet, l'emploi généralisé de la moustiquaire entraine une baisse considérable de la transmission du paludisme de l'ordre de $90 \%$ (Carnevale et al., 1991). A Yabassi, la transmission du paludisme est assurée par An. gambiae et An. coluzzii. An. gambiae apparait comme le vecteur majeur pendant la période d'étude. Les résultats similaires faisant de cette espèce le vecteur principal du paludisme dans la forêt dégradée du sud-Cameroun ont déjà été trouvés (Akono et al., 2014 ; 2015). Par ailleurs, aucun spécimen d'An. nili et $d^{\prime \prime} A n$. ziemanni n'a été trouvé infecté par $P$. falciparum dans l'échantillon collecté. Pourtant certaines études relevant les rôles joués par ces deux espèces dans quelques facies écologiques au Cameroun ont été menées (Carnevale et al., 1992; Tabue et al., 2014). L'absence dans nos résultats des spécimens infectés appartenantà ces deux espèces peut être due au faible effort d'échantillonnage dans nos sites d'études. Ces espèces auraient donc une implication négligeable dans la transmission du paludisme tel que relevés par certains auteurs (Antonio-Nkondjio et al., 2006).

les populations locales à choisir les meilleures méthodes préventive et curative en vigueur pour se protéger du paludisme qui demeure une réalité dans les sites d'étude, particulièrement à Yabassi. 


\section{REMERCIEMENTS}

Les auteurs remercient les populations de BODIMAN et YABASSI pour leur collaboration.

\section{REFERENCES}

Akono NP, Tonga C, Kekeunou S, Lehman LG, 2014. Mosquito species diversity and malaria transmission in Ayos, an area of degraded forest targeted for universal long-lasting insecticidal net distribution in southern Cameroon. African Entomology 22: 602-610.

Akono NP, Tonga C, Mbida Mbida J A, Ngo Hondt OE, Awono Ambene H P, Ndo C, Magne Tamdem G, Peka MF, Ngaha R, Lehman LG, 2015. Anopheles gambiae, vecteur majeur $\mathrm{du}$ paludisme à Logbessou, zone péri-urbaine de Douala (Cameroun). Bulletin de la Société Pathologique et Exotique 108:360-368.

Antonio-Nkondjio C, Kerah CH, Simard F, AwonoAmbene $P$, Chouaibou M, Tchuinkam T, Fontenille D, 2006. Complexity of malaria vectorial system in Cameroon: contribution of secondary vectors to malaria transmission. Journal of Medical Entomology 43:1215-1221.

Antonio-Nkondjio C, Defo-Talom B, Tagne-Fotso R, TeneFossog B, Ndo C, Lehman L G, Tchuinkam T, Kengne $P, \quad$ Awono-Ambene $P, 2012$. High mosquito burden and malaria transmission in a district of the city of Douala, Cameroon. BMC Infectious Diseases 12:275.

Burkot T R, Williams J L, Schneider I, 1984. Identification of Plasmodium falciparum-infected mosquitoes by a double antibody enzyme-linked immunosorbent assay. The American Journal of Tropical Medicine and Hygiene 33: 783-788.

Carnevale P, Mouchet J, 2001. «La lutte antivectorielle au Cameroun. Passé-présent- avenir. Réflexions». Bulletin de la Société Pathologique et Exotique 94:202-209.

Carnevale P, Le Goff G, Toto J C, Robert V, 1992. Anopheles nili as the main malaria vector in villages of Southern Cameroon. Medical Veterinary Entomology 6: 135-138.

Carnevale P, Robert V, Snow R, Curte C, Richard A, Boudin C, Pazart L H, Halna J M, Mouchet J, 1991. L'impact des moustiquaires imprégnées sur la prévalence et la morbidité liée au paludisme en Afrique sub-saharienenne. Annales de la Société Belge de Médecine Tropicale 71: 127-150.

Edwards W, 1941. Clé des Culicinae adultes de la région éthiopienne. ORSTOM., Laboratoire Entomologie Médicale. Snoopy. BondyTraduction de Rageau.

Etang J, Mbida A, Akono NP, Binyang J, Moukoko ECE, Lehman LG, Awono-Ambene P, Talipouo A, Ekoko WE, Tagne D, Tchoffo R, Manga L, Mimpfoundi R, 2016. Anopheles coluzzii larval habitat and insecticide resistance in the island area of Manoka, Cameroon. BMC Infectious Diseases 16:217.

Gillies MT, De Meillon B, 1968. The Anophelinae of Africa South of the Sahara. South African Institute of Medical Research 54:1-343.

Gillies MT, Coetzee M, 1987. Supplement to the Anophelinae of Africa South of the Sahara. Publications of the South African Institute of Medical Research Johannesburg, no. 55.

Goïta A, 2010. Connaissances, attitudes et pratiques des populations face au paludisme dans l'aire de santé de Baguinéda. Thèse de Doctorat, Université de Bamako, pp 1-124.

Hamon J, Abonnenc $E$, Noel $E, 1955$. Contribution à l'étude des culicidés de l'ouest du Sénégal. Annales de Parasitologie Humaine et Comparée $30: 278-308$.

Kelani R, Aïtchedji M, Yadouléton A, Allagbé H, Issaou G, Degla S, 2014. Utilisation des moustiquaires imprégnées à Longue Durée d'action (MILD) au Bénin contre le paludisme: impacts des pratiques de lavage en milieu communautaire sur leur efficacité. International Journal of Innovation and Applied Studies 7:1310-1320.

Lindsay SW, Snow RW, Broomfield G L, Semega JM, Wirtz RA, Greenwood BM, 1989. Impact of permethrin-treated bednets on malaria transmission by the Anopheles gambiae complex in The Gambia. Medical Veterinary Entomology 3:263-71.

Lines JD, Myamba J, Curtis CF, 1987. Experimental hut trials of permethrin-impregnated mosquito nets and eave curtains against malaria vectors in Tanzania. Medical Veterinary Entomology 1:3751.

Minsanté (Ministère de la santé publique du Cameroun), 2008. Cameroun : vers un meilleur accès à la prévention et au traitement du SIDA, de la 
tuberculose et du paludisme. [http:// www.afriscoopnet/journal/spipphp].

OMS (Organisation Mondiale de la Santé), 2014. Entomologie du paludisme et lutte antivectorielle, pp 1- 200.

Rickenbach A, Ferrara L, Eouzan J P, Germain M, Button $\mathrm{J}$, 1972. Cycle d'agressivité et répartition verticale de quelques espèces de moustiques forestiers de la région de Yaoundé (Cameroun). Cahiers O.R.S.T.O.M., Série Entomologie Médicale et Parasitologie 10:309-325.

Santolamazza F, Mancini E, Simard F, Qi Y, Tu Z, Della Torre A, 2008. Insertion polymorphisms of SINE200 retrotransposons within speciation islands of Anopheles gambiae molecular forms. Malaria Journal 7:163.

Seck I, Fall IS, Faye A, Ba O, Tal-Dia A, 2008. « Connaissances, attitudes et pratiques des femmes sur le paludisme, dans la zone rurale de Poponguine, Sénégal ». Médecine Tropicale 68:629-633.

Tabue RN, Nem T, Atangana J, Bigoga JD, Patchoke S, Tchouine F, Fodjo BY, Leke R G F, Fondjo E, 2014. Anopheles ziemanni a locally important malaria vector in Ndop health district, north west region of Cameroon. Parasites and Vectors 7:262.

Tene Fossog B, Ayala D, Acevedo P, Kengne P, Ngomo
IAM, Makanga B, Magnus J, Awono-Ambene P, Njiokou F, Pombi M, Antonio-Nkondjio C, Paupy C, Besansky NJ, Costantini C, 2015. Habitat segregation and ecological character displacement in cryptic African malaria mosquitoes. Evolutionary Applications 8: 326345.

Tene Fossog B, Antonio-Nkondjio C, Kengne P, Njiokou F, Besansky NJ, Costantini C, 2013. Physiological correlates of ecological divergence along an urbanization gradient: differential tolerance to ammonia among molecular forms of the malaria mosquito Anopheles gambiae. BMC Ecology 13:1.

Toto JC, Besnard P, Le Mire J, Almeida DSI, Dos Santos MA, Fortes F, Foumane V, Simard F, Awono Ambene HP, Carnevale P, 2011. Premiers tests OMS d'évaluation de la sensibilité aux insecticides chez Anopheles gambiae et Culex quinquefasciatus à Lobito, Angola. Bulletin de la Société Pathologique et Exotique 104:307-312.

Zoulani A, Carnevale P, Penchenier L, 1994. Influence des moustiquaires imprégnées de deltaméthrine sur le cycle d'agressivité d'Anopheles gambiae à Djoumouna, Congo. Annales de la Société Belge de Médecine Tropicale74 (2) :83-91. 